\title{
Comparison of stroke volume measurement between non-invasive bioreactance and oesophageal Doppler in patients undergoing major abdominal-pelvic surgery
}

Gennaro De Pascale, Mervyn Singer, David Brealey

Division of Medicine, Bloomsbury Institute of Intensive Care Medicine, University College Hospital, Gower St, London, WC1E 6BT, UK.

Division of Anaesthesia, University College Hospital, 235 Euston Road, London, NW1 2BU, UK

Short Title: Bioreactance technology vs. Oesophageal Doppler in major surgery

Funding: No funding was received from either Deltex (CardioQ manufacturer) or Cheetah (NICOM manufacturer) for this study. NICOM electrodes were provided free for this evaluation by Cheetah.

Conflicts of interest: Mervyn Singer sits on an advisory board for Deltex; his academic fund at UCL receives an annual unrestricted donation from Deltex. UCL also hold shares in Deltex donated by the Pauline Thomas Charitable Trust. He was not involved in the collection of data for this study.

Acknowledgments Authors' contribution:

$\operatorname{Dr}$ De Pascale takes responsibility for the content of the manuscript, including the data and analysis.

Dr De Pascale, Dr Brealey contributed to conception and design, acquisition of data, or analysis and interpretation of data; drafted the submitted article or revised it critically for important intellectual content and provided final approval of the version to be published

Prof Singer contributed to the design of the design, revised critically the paper for important intellectual content and provided final approval of the version to be published

Correspondence to: Dr Gennaro De Pascale , MD, Policlinico Universitario A. Gemelli Università Cattolica del Sacro Cuore Largo A. Gemelli, 8 , 00168 Rome-Italy . Tel.+ 390630154889 (ICU) + 390630155886 (office). Fax: + 39063013 450. Email: gennaro.depascalemd@gmail.com 


\section{Abstract}

Background: Bioreactance technology has been recently proposed as a novel, non-invasive technology for measuring stroke volume(SV) and cardiac output in the operating room and critical care setting. We set out to evaluate how the $\mathrm{NICOM}^{\circledR}$ bioreactance device performed against the CardioQ oesophageal Doppler monitor in patients undergoing major abdominal-pelvic procedures.

Methods: $\mathrm{SV}_{\text {Nicom }}$ and $\mathrm{SV} \mathrm{VDM}_{\mathrm{O}}$ were simultaneously measured intraoperatively, including before and after clinical interventions such as, fluid challenge, vasopressor boluses, peritoneal gas insufflation/removal, and Trendelenburg/reverse Trendelenburg patient positioning.

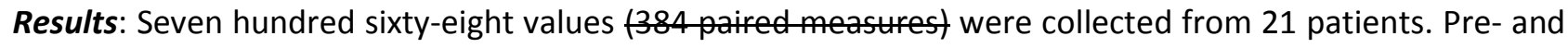
post-intervention measures were recorded on 155 occasions. Bland-Altman analysis revealed a bias of 8.6 $\mathrm{ml}$ and poor precision with wide limits of agreement $(54 \mathrm{ml}$ and $-37 \mathrm{ml}$ ) and a percentage error of $50.6 \%$. No improvement in precision was detected after taking into account repeated measurements for each patient (bias: $8 \mathrm{ml}$; limits of agreement: $74 \mathrm{ml}$ and $-59 \mathrm{ml}$ ). Concordance between changes in $\mathrm{SV}_{\text {Nicom }}$ and SV $V_{\text {ODM }}$ before and after interventions was also poor: $78.7 \%$ (all measures), $82.4 \%$ (after vasopressor administration) and $74.3 \%$ (after fluid challenge). Using Doppler SV as the reference technique, the area under the receiver operating characteristic curve constructed to assess the ability of the NICOM device to predict fluid responsiveness was $0.81(0.7-0.9)$.

Conclusions: In patients undergoing major abdominopelvic surgery, SV values obtained by NICOM did not show either clinically or statistically acceptable agreement with those obtained by oesophageal Doppler. In the setting of this study, bioreactance technology cannot reliably replace oesophageal Doppler monitoring. Trial registration: Observational Study

Key words: Bioreactance technology. Oesophageal doppler. Haemodynamic monitoring 


\section{Introduction}

Perioperative haemodynamic optimisation has been shown to improve postoperative outcomes following major surgery ${ }^{1,2}$. Various strategies have been adopted including stroke volume (SV) optimization by fluid challenges, or targeting a minimum tissue oxygen delivery value using fluid \pm inotropic support. Pulmonary artery catheterization has now been largely supplanted by a variety of minimally or non-invasive haemodynamic monitoring techniques ${ }^{3}$, albeit with varying strengths of validation.

On the basis of its evidence base, oesophageal Doppler flowmetry was the haemodynamic monitoring modality recommended for perioperative optimization by the National Institute for Health and Clinical Excellence (NICE) (www.nice.org.uk). This technique measures the velocity of blood within the descending thoracic aorta using an oesophageal probe sited at the level of the fifth to sixth thoracic vertebra emitting 4 $\mathrm{MHz}$ Doppler ultrasound. The area under the velocity-time waveform is the stroke distance, i.e. the distance a column of blood travels down the aorta with each left ventricular ejection. Using a formula incorporating the patient's age, height and weight, the descending aortic stroke distance can be translated into a reasonably accurate measure of left ventricular stroke volume ${ }^{4,5}$. This has been validated in multiple studies $^{6}$. Necessary assumptions are that fluctuations in blood pressure, body temperature and cardiac output do not affect the ratio of upper to lower body blood flow?

The Non-Invasive Cardiac Output Monitoring (NICOM) utilizes the bio-reactance. This technique is an evolution of bioimpedance and is based on the analysis of intra-beat variations in voltage phase shifts. It is totally non-invasive, requiring only the application of four electrodes onto the chest surface ${ }^{8}$. NICOM has been successfully used in adult and paediatric settings, especially as a tool for predicting fluid responsiveness ${ }^{9,10}$. However, recent investigations comparing this device against different reference monitoring techniques, including oesophageal Doppler, in either the operating or emergency settings, have 
shown conflicting results ${ }^{11}, 12$. The aim of this evaluation was to assess the validity and precision of stroke volume (SV) measurements using the bioreactance device (NICOM) compared against oesophageal Doppler (ODM) during major elective abdomino-pelvic surgery with particular emphasis on the impact of specific interventions such as fluid challenge, vasopressor administration, peritoneal gas insufflation/removal, and Trendelenberg/reverse Trendelenberg positioning.

\section{Methods}

\section{Patients and study design}

The results presented in this paper were obtained as part of a device evaluation at University College London Hospitals. The monitoring, interventions and data collected were part of standard care. In addition, the analysed data were fully anonymised and, as such, specific ethics committee permission was not required. During the study period ( $1^{\text {st }}$ November 2014 through $31^{\text {st }}$ January 2015), all consecutive patients undergoing a major abdominal procedure (either laparoscopic or open) were considered eligible whenever intra-operative cardiac output (CO) and invasive arterial pressure monitoring were scheduled. Patients were enrolled if in addition to standard cardiac output monitoring i.e. using oesophageal Doppler [CardioQ, Deltex Medical, Chichester, UK] the attending consultant anaesthetist decided to evaluate the NICOM (Cheetah Medical, Portland, Oregon, USA) as a completely non-invasive cardiac output monitoring tool. Subjects were excluded if they had any skin abnormalities or if they had a contraindication for the use of oesophageal Doppler monitoring (i.e. known or suspected oesophageal ulcer, malformation, varicose, or tumour). All patients underwent general anaesthesia, they were induced with propofol and fentanyl, whilst desflurane in oxygen enriched air was used for maintenance. The lungs were mechanically ventilated via an endotracheal tube at a tidal volume of $6-8 \mathrm{ml} / \mathrm{kg}$ ideal body weight. Normothermia was maintained using a whole-body convective warming blanket and a fluid warmer. Epidural analgesia and central venous pressure monitoring were performed where indicated. After surgery patients were moved to either the 
recovery room or a high dependency area as clinically needed. The NICOM ${ }^{\circledR}$ system was calibrated before the induction of anaesthesia. Four proprietary dual skin electrodes were placed over the left and right clavicles, and on the left and right lower thorax at the level of the diaphragm. Local body hair was shaved prior to placement, and the site cleaned with a chlorhexidine $2 \%$ sponge. A waterproof dressing was then applied over each dual electrode. One of the electrodes transmits a high frequency current across the body, whereas the other connects to a voltage amplifier. Signals are applied to, and recorded from, both the left and right side. The NICOM was calibrated to average stroke volume over 30 seconds. Electrodes were removed shortly after the end of surgery. After the induction of anaesthesia and tracheal intubation, the CardioQ oesophageal Doppler device was set up. A probe was inserted into the oesophagus to the midthoracic level, to a depth of approximately $35-40 \mathrm{~cm}$ from the teeth (or $5 \mathrm{~cm}$ further if inserted nasally). The probe position was then adjusted using visual and audible cues to obtain the highest Doppler frequency shift signal from blood flow in the thoracic descending aorta. The probe position was re-adjusted if signal quality deteriorated. Simultaneous snapshots of stroke volume were obtained from both devices every 5-15 minutes. Other collected data included cardiac output/index, mean arterial pressure, central venous pressure (when available), heart rate, and peak blood flow velocity and flow time corrected for heart rate (oesophageal Doppler only). Two investigators (GDP, DB) evaluated the obtained waveforms and discarded any appearing as poor quality or artefactual. Patient haemodynamic status was recorded prior to and each minute after an intervention until the maximum effect was observed. Interventions included fluid challenges with either colloid or crystalloid (250-500 mL > $10 \mathrm{~min}$ ) which were repeated if the patient was fluid responsive, vasopressor administration (metaraminol $0.5-1 \mathrm{mg}$ bolus followed by norepinephrine infusion $0.5 \mathrm{mcg} / \mathrm{kg} / \mathrm{min}$ or higher, if the patient was not responsive), peritoneal gas insufflation/removal (for laparoscopic procedures), and Trendelenburg/reverse Trendelenburg positions (robotic surgery and laparoscopic procedures) 


\section{Data analysis}

The Kolmogorov-Smirnov test was used to evaluate variable distribution. Data with a non-normal distribution were assessed with the Mann-Whitney test with median and 25th to 75th centile values reported. Normally distributed data were assessed with Student's t-test. Categorical variables are presented as proportions. A p value below 0.05 was considered significant. Bland-Altman plots (mean stroke volume measurement from the two devices versus the difference in measurements between the devices) were used to calculate precision and bias. We determined a priori that a $30 \%$ error was acceptable, as recommended by Critchley and Critchley ${ }^{13,14}$. The percentage error was calculated as 2SD divided by the mean of $S V_{O D M}$. Concordance was assessed by plotting the test $\Delta \mathrm{SV}_{\text {Nicom }}$ against the reference $\Delta S V_{\text {ODM }}$ on a four-quadrant scatter plot $^{15}$. The concordance rate is the percentage of the number of data points lying in the upper right and the lower left quadrant of the scatter plot in relation to the total number of data points. Data at the center of the plot represent only small and random changes and hence were excluded from analysis (exclusion zone). Using recommendations recently proposed by Critchley et al, we applied an exclusion zone of $15 \%$, with sufficient concordance to assume interchangeability $>90 \%{ }^{15}$. For testing the ability of the changes in $\mathrm{SV}_{\text {Nicom }}$ induced by a fluid challenge to predict fluid responsiveness, we constructed receiver operating characteristics (ROC) curves. Sensitivity and specificity are expressed as median ( $95 \%$ confidence interval). Values considerate inaccurate were discarded and not included in the analysis.MedCalc ${ }^{\circledR}$ Software (v. 12.2.1, MariaKerke, Belgium) was used for all statistical analyses. Graphing of data was undertaken using Prism version 6.0 for Windows (graphPad Software, San Diego, CA). 


\section{Results}

Patients

The devices were evaluated in 21 patients whose characteristics are described in Table 1. The mean age of the cohort was $65 \pm 12$ years and were predominantly male $(15 / 21,71.4 \%)$. Main comorbidities were chronic cardiovascular disease, chronic respiratory disease, diabetes and obesity: $81 \%$ of the patients were classified as ASA (American Society of Anaesthesiologists) 2 or 3. Six patients underwent laparoscopic robotic procedures (five prostatectomies, one cystectomy) and 15 open complex major abdominal procedures. Mean \pm SD intraoperative fluid balance was $+1564 \pm 949 \mathrm{ml}$ and half of the patients were admitted to ICU for post-operative care.

A total of 471 paired measures were obtained from the 21 patients. Eighty-seven measures were discarded as they were deemed inaccurate by the investigators - unreliable waveforms for oesophageal Doppler $(n=36)$ and electrical cautery interference for NICOM $(n=51)$. These inaccurate measures mainly occurred during the initial and final surgical phase. Thus 384 paired measurements (median [min-max] per patient: 16 [6-34]) have been included in the analysis.

One hundred and fifty-five paired SV values were collected before and after a standard intervention that would likely induce a haemodynamic change - 66 fluid challenges, 68 boluses or infusions of vasopressor, 11 laparoscopic gas insufflations/ removals, and 10 position changes.

Comparison of absolute values of $S V_{O D M}$ and $S V_{\text {Nicom }}$

Considering all 384 paired measures, the bias between SV $\mathrm{ODM}_{\text {M }}$ and SVNicom was $8.6 \mathrm{ml}$ with precision (limits of agreement) of $54.3 \mathrm{ml}$ and $-37.1 \mathrm{ml}$ and a percentage error of $50.6 \%$ (Figure 1). When analysed with a Bland-Altman plot, taking into account repeated measurements for each patient, the bias between SV $\mathrm{ODM}_{\mathrm{M}}$ and $\mathrm{SV}_{\text {Nicom }}$ was $7.5 \mathrm{ml}$, with wider limits of agreement $(74 \mathrm{ml}$ and $-59 \mathrm{ml})$. 
The Bland-Altman analysis performed according to the use of epidural analgesia showed similar results: in patients with epidural, the bias between SV $V_{O D M}$ and SVNicom was $9.7 \pm 20.9 \mathrm{ml}$ with precision (limits of agreement) of $50.6 \mathrm{ml}$ and $-31.3 \mathrm{ml}$ and a percentage error of $44.2 \%$; in patients without epidural, the bias between SV $V_{O D M}$ and SVNicom was $9.7 \pm 26.4 \mathrm{ml}$ with precision (limits of agreement) of $58.9 \mathrm{ml}$ and $-44.6 \mathrm{ml}$ and a percentage error of $59.7 \%$.

\section{Comparison of changes in SVODM and $S V_{\text {Nicom }}$}

The concordance proportion between changes in SV $\mathrm{ODM}_{\mathrm{O}}$ and $\mathrm{SV}_{\text {Nicom }}$ induced by any type of intervention $(n=$ 155) was $78.7 \%$. Excluding changes $<15 \%$, the concordance rate was $73 \%$ (Figure $2 a$ ). The concordance proportion between changes in $\mathrm{SV}_{\mathrm{ODM}}$ and $\mathrm{SV}_{\text {Nicom }}$ induced by bolus vasopressor administrations/infusion ( $n=68$ ) was $82.4 \%$; excluding changes $<15 \%$ the concordance proportion was $82.1 \%$ (Figure $2 b$ ).

The concordance proportion between changes in $\mathrm{SV}_{\mathrm{ODM}}$ and $\mathrm{SV}_{\text {Nicom }}$ induced by fluid challenge administrations $(n=66)$ was $74.3 \%$; excluding changes $<15 \%$ the concordance proportion was $68.6 \%$ (Figure 2c).

Ability of $N_{I C O M}{ }^{\circledR}$ device to assess fluid responsiveness

After a fluid challenge, an increase in $\mathrm{SV}_{\text {Nicom }}>10 \%$ predicted fluid responsiveness with a sensitivity of $80 \%$ $(56.3-94.3)$ and specificity of $82.6 \%(68.6-92.2)$. The area under the ROC constructed to assess the ability of the NICOM device to predict fluid responsiveness was $0.81(0.7-0.9)$ (Figure 3). 


\section{Discussion}

In this cohort of patients undergoing elective abdomino-pelvic operations, SV values obtained by NICOM did not show acceptable agreement, either clinically or statistically, against those obtained simultaneously with oesophageal Doppler. The ability of NICOM to detect changes in SV after standard interventions capable of inducing haemodynamic changes was also suboptimal. However its accuracy in predicting fluid responsiveness was higher, with a sensitivity of $80 \%$ and a specificity of $82.6 \%$.

The NICOM device utilizes bioreactance technology which is based on voltage shifts occurring when alternating electrical currents, passing through the thorax, are affected by pulsatile blood flow ${ }^{8}$. As it is completely non-invasive, safe and very simple to use, this device does appear attractive, especially for specialties such as paediatrics ${ }^{16}$. Its application has recently extended to adult patients in the emergency department and operating theatres, where more invasive, well validated monitoring systems are widely used $^{17}$. We used an oesophageal Doppler as our reference method for SV measurement as this is the standard device used perioperative within University College Hospital. Oesophageal Doppler is widely accepted as a reliable method for cardiac output monitoring in clinical practice and has been validated against thermodilution and other techniques in multiple studies ${ }^{6}$. It is easy to use and allows continuous haemodynamic monitoring by on-line analysis of the features of the descending aortic blood flow velocity waveform.

In this study, considering all paired measures from the entire cohort, the agreement between the two monitoring systems was poor, with an error percentage much higher than the accepted $30 \%$ cut-off. We also evaluated concordance between the two methods by using repeated measures, performing BlandAltman analysis accounting for different repeated measurements for each patient. However, taking into account the presence of a proportional error, the disagreement between the monitoring devices did not decrease. Most of the clinical data supporting the accuracy of bioreactance in adults derives from intra- 
and post-operative cardiac surgery, ${ }^{9,18}$. Recent studies comparing NICOM against oesophageal Doppler flowmetry have generated conflicting results. Waldron et al compared NICOM and CardioQ devices for guiding goal-directed fluid therapy (GDFT) during colorectal surgery: although agreement in assessing response to a fluid challenge was poor $(60-66 \%)$, they found no difference in clinical outcomes ${ }^{19}$. On the other hand, Conway et al also studied patients undergoing major, open abdominal surgery, and reported a bias of $-6.9 \mathrm{ml}$, wide limits of agreement $(-22.9$ to $36.8 \mathrm{ml})$ and a percentage error of $57 \%$. Both devices delivered unacceptable measures during electrical diathermy ${ }^{11}$. In another intraoperative study, Huang et al compared NICOM simultaneously against both suprasternal and oesophageal Doppler techniques. They observed that the trending ability of NICOM was less reliable, and was significantly influenced during upper abdominal interventions (e.g. open retractor placement, laparoscopic insufflation and head-down tilt) with unpredictable shifts in cardiac output $>1 \mathrm{~L} / \mathrm{min} / \mathrm{m}^{220}$. The observations made by Huang et al are in line with our data where cautery interference was significant for both monitors ( $18.5 \%$ of all collected readings). In such conditions, NICOM latency, based on the minimum sample interval value of at least 30 seconds, resulted in slower changes in SV than oesophageal Doppler.

Overall, we found suboptimal agreement $(78.7 \%)$ between the two devices in tracking change in stroke volume. Exclusion of changes $<15 \%$ did not improve upon this agreement. Specific non-fluid interventions (e.g. vasopressors or postural change) were separately associated with suboptimal agreement. The overall accuracy of the NICOM device to predict fluid responsiveness was better, at approximately $81 \%$, with comparable sensitivity and specificity.

Reasons for this disparity include a possible influence of current flux pathways related to the position of the lower chest NICOM electrodes as the dermatome level was not precisely identified. Changes in geometry of the upper abdomen related to the interventions may also affect the bioreactance readings. The oesophageal Doppler technique assumes a fixed descending aortic diameter during systole and proportionality maintained between upper and lower body blood flow. These assumptions may not hold 
true with the varied interventions given, though Huang et al did report good correlation between suprasternal and oesophageal Doppler techniques.

This evaluation has limitations. First, no 'gold standard' technique was used as a comparator. Traditionally, thermodilution cardiac output measured by invasive pulmonary artery catheterisation has been used for comparison studies. However, this technique is far from perfect ${ }^{21,22}$ and is now very rarely used at our centre. Second, we only included patients undergoing elective abdominal-pelvic procedures and our findings should not be extrapolated to other settings or to patients in intensive care. Thirdly, we did not address the role of NICOM calibration shifts during specific phases of the surgical intervention as a potential determinant of the suboptimal concordance with oesophageal Doppler. We were not blinded to the therapeutic interventions. Further, due to the design of the study, we were not able to specifically analyse the time-course of patients' haemodynamic status and the clinical impact of the observed measures. Finally, this was not a clinical trial where interventions would have been standardised and, due to the observational nature of the investigation, a selection bias could not be avoided. However, these devices were being evaluated in a real world clinical setting and how they would be used if adopted into practice at our institution. To date, our population is the largest in which interventions other than fluid, have been specifically used to compare the CardioQ and NICOM devices.

\section{Conclusions}

In patients undergoing major surgery under general anaesthesia, stroke volume values obtained by NICOM showed an unacceptably high percentage error compared to those obtained with oesophageal Doppler. Trending ability following varied interventions was suboptimal; the observed accuracy for predicting fluid responsiveness was approximately $80 \%$. 


\section{Key messages}

- In patients undergoing major procedures, high percentage error of NICOM device compared with oesophageal Doppler was observed

- Concordance between SV changes after different kind of interventions was also suboptimal, whereas the accuracy in predicting fluid responsiveness was higher.

- In the setting of this study, NICOM device and oesophageal Doppler are not interchangeable 


\section{References}

1. Shoemaker WC, Appel PL, Kram HB, Waxman K, Lee TS. Prospective trial of supernormal values of survivors as therapeutic goals in high-risk surgical patients. Chest 1988; 94:1176-86.

2. Boyd O, Grounds RM, Bennett ED. A randomized clinical trial of the effect of deliberate perioperative increase of oxygen delivery on mortality in high-risk surgical patients. JAMA 1993; 270:2699-707.

3. Saugel B, Cecconi M, Wagner JY, Reuter DA. Non-invasive continuous cardiac output monitoring in perioperative and intensive care medicine. Br J Anaesth 2015; 114:562-75.

4. Singer M. Oesophageal Doppler monitoring: should it be routine for high-risk surgical patients? Curr Opin Anaesthesiol 2011; 24:171-6.

5. Singer M. Oesophageal Doppler. Curr Opin Crit Care 2009; 15:244-8.

6. Dark PM, Singer M. The validity of trans-oesophageal Doppler ultrasonography as a measure of cardiac output in critically ill adults. Intensive Care Med 2004; 30:2060-6.

7. Tomlin PJ, Duck FA. Transoesophageal aortic velography in man. Can Anaesth Soc J 1975 ; 22:56171.

8. Raval NY, Squara P, Cleman M, Yalamanchili K, Winklmaier M, Burkhoff D. Multicenter evaluation of noninvasive cardiac output measurement by bioreactance technique. J Clin Monit Comput 2008; 22:113-9.

9. Marqué S, Cariou A, Chiche JD, Squara P. Comparison between Flotrac-Vigileo and Bioreactance, a totally noninvasive method for cardiac output monitoring. Crit Care 2009; 13:R73.

10. Squara P, Rotcajg D, Denjean D, Estagnasie P, Brusset A. Comparison of monitoring performance of Bioreactance vs. pulse contour during lung recruitment manoeuvres. Crit Care 2009; 13:R125.

11. Conway DH, Hussain OA, Gall I. A comparison of noninvasive bioreactance with oesophageal Doppler estimation of stroke volume during open abdominal surgery: an observational study. Eur J 
Anaesthesiol 2013; 30:501-8.

12. Kupersztych-Hagege E, Teboul JL, Artigas A et al. Bioreactance is not reliable for estimating cardiac output and the effects of passive leg raising in critically ill patients. Br J Anaesth 2013; 111:961-6.

13. Critchley LA, Critchley JA. A meta-analysis of studies using bias and precision statistics to compare cardiac output measurement techniques. J Clin Monit Comput 1999;15:85-91.

14. Bland JM, Altman DG. Measuring agreement in method comparison studies. Stat Methods Med Res 1999; 8:135-160.

15. Critchley LA, Lee A, Ho AM. A critical review of the ability of continuous cardiac output monitors to measure trends in cardiac output. Anesth Analg 2010; 111:1180-92.

16. Weisz DE, Jain A, McNamara PJ, EL-Khuffash A. Non-invasive cardiac output monitoring in neonates using bioreactance: a comparison with echocardiography. Neonatology 2012; 102:61-7.

17. Dunham CM, Chirichella TJ, Gruber BS et al. Emergency department noninvasive (NICOM) cardiac outputs are associated with trauma activation, patient injury severity and host conditions and mortality. J Trauma Acute Care Surg 2012; 73:479-85.

18. Cheung $\mathrm{H}$, Dong $\mathrm{Q}$, Dong $\mathrm{R}, \mathrm{Yu}$ B. Correlation of cardiac output measured by non-invasive continuous cardiac output monitoring (NICOM) and thermodilution in patients undergoing offpump coronary artery bypass surgery. J Anesth 2015; 29:416-20.

19. Waldron NH, Miller TE, Thacker JK et al. A prospective comparison of a noninvasive cardiac output monitor versus esophageal Doppler monitor for goal-directed fluid therapy in colorectal surgery patients. Anesth Analg 2014; 118:966-75.

20. Huang L, Critchley LA, Zhang J. Major Upper Abdominal Surgery Alters the Calibration of Bioreactance Cardiac Output Readings, the NICOM, When Comparisons Are Made Against Suprasternal and Oesophageal Doppler Intraoperatively. Anesth Analg 2015; 121:936-45.

21. Cecconi M, De Backer D, Antonelli $M$ et al. Consensus on circulatory shock and hemodynamic 
monitoring. Task force of the European Society of Intensive Care Medicine. Intensive Care Med 2014; 40:1795-815.

22. Cecconi M, Arulkumaran N, Kilic J, Ebm C, Rhodes A. Update on hemodynamic monitoring and management in septic patients. Minerva Anestesiol;80:701-711. 
Figure 1

Bland-Altman analysis for stroke volume (SV) measurements by oesophageal Doppler (ODM) and bioreactance (NICOM) for all paired data values ( $n=384)$.

Bias: $8.6 \pm 23.3$

LOA: 91.4

PE: $50.6 \%$

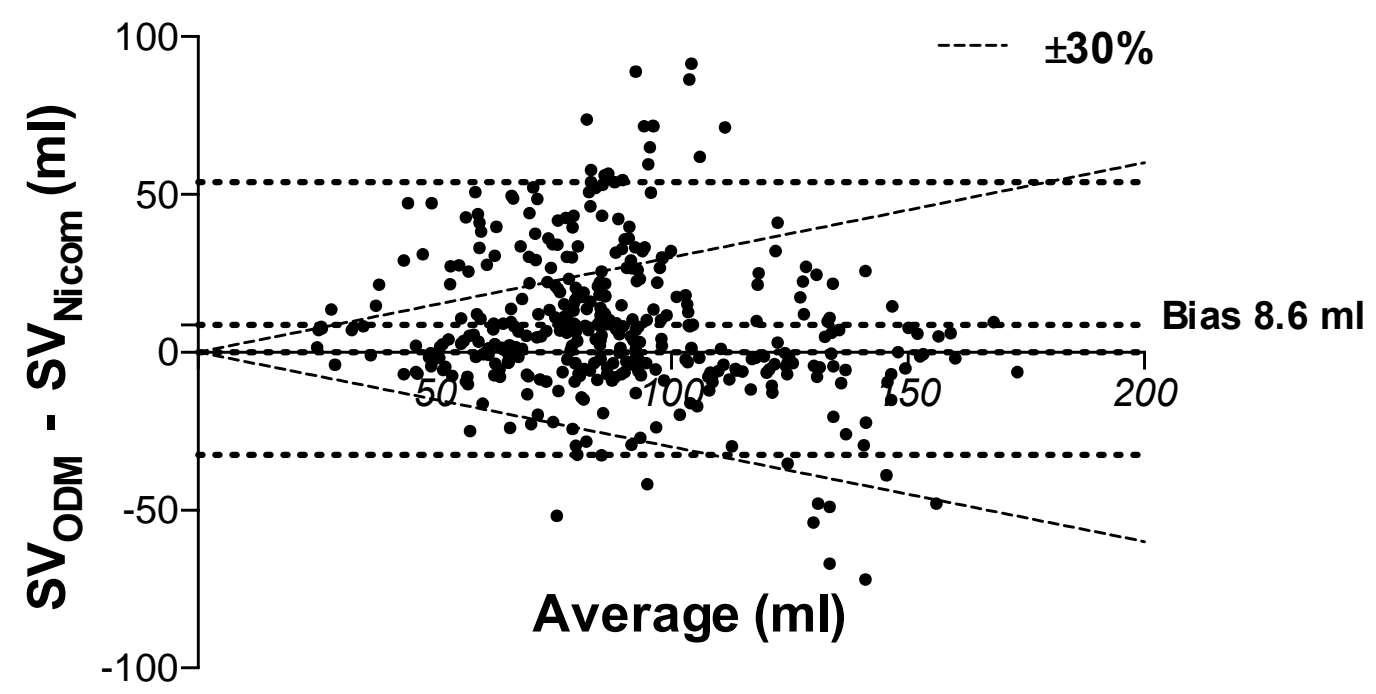

Limits of agreement, LOA; PE, percentage error. 
Figure 2: Concordance proportions between NICOM and oesophageal Doppler.

(a) Concordance proportion with and without an exclusion zone of $15 \%$ (any intervention type, $n=155$ )

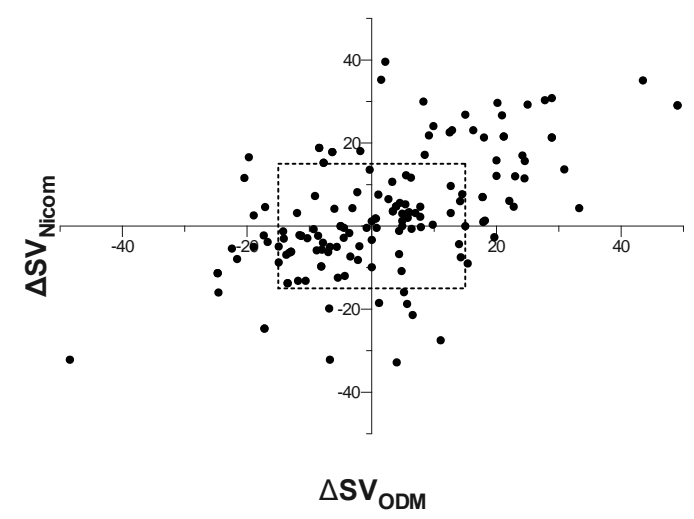

(b) Concordance proportion with and without an exclusion zone of $15 \%$ (bolus vasopressors administrations, $n=68$ )

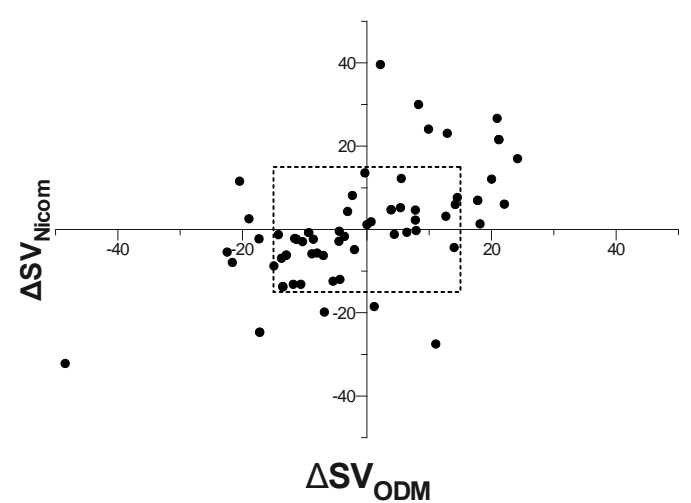

(c): Concordance proportion with and without an exclusion zone of $15 \%$ (fluid challenges, $n=66$ )

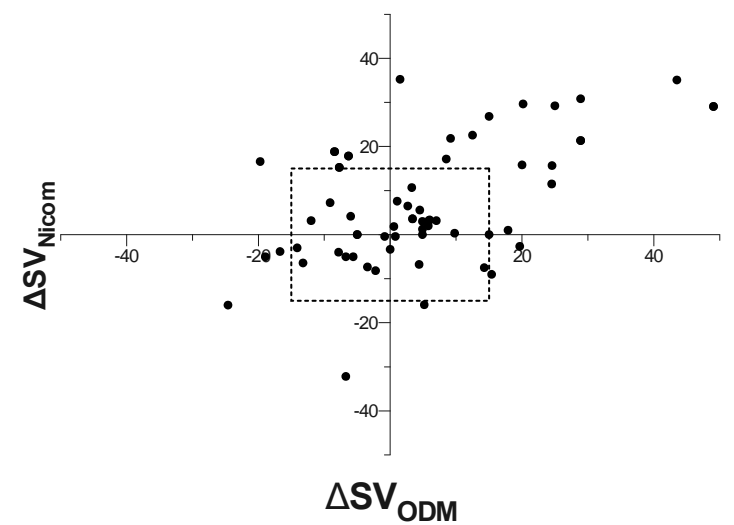

SV, stroke volume; ODM, oesophageal Doppler. 
Figure 3: ROC curve testing the ability of the NICOM device, using ODM as reference method, to predict fluid responsiveness using the percentage change in $\mathrm{SV}\left(\mathrm{SV}_{\text {Nicom }}\right)$ induced by fluid challenges.

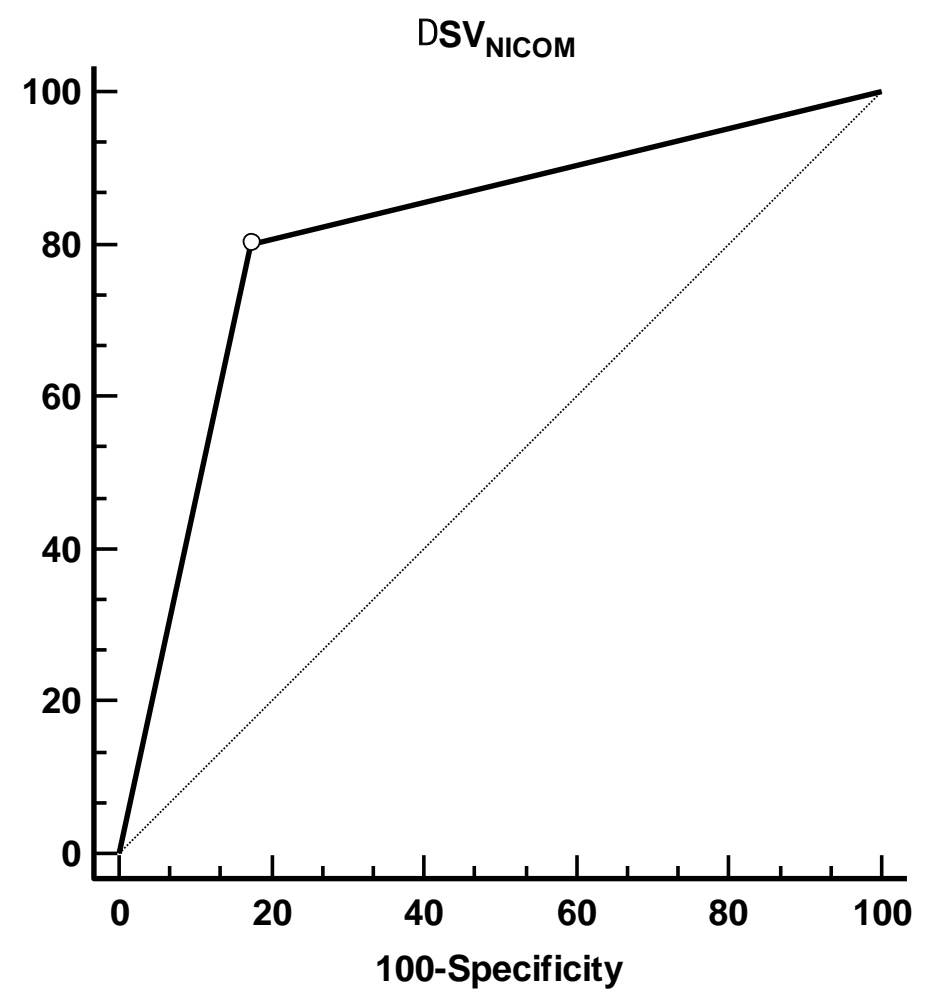

DSV NICOM $_{\text {Delta-SV }}$ NICOM; ODM Oesophageal Doppler 
Table 1 Patient characteristics

VARIABLE

RESULTS

\begin{tabular}{lc}
\hline Patients, (n) & 21 \\
\hline Age, years & $15(71.4 \%)$ \\
\hline Male sex, $\mathbf{n}(\%)$ & $1.9 \pm 0.2$ \\
\hline BSA, $\mathbf{m}^{2}$ & $27 \pm 9.2$ \\
\hline BMI Kg/cm & \\
\hline Patients with ASA physical status II/III, $\mathbf{n}$ (\%) & $17(80.6)$ \\
\hline Intraoperative fluid balance, $\mathbf{m l}$ & $1564 \pm 949$ \\
\hline Duration of surgery, min & $164 \pm 54$ \\
\hline Epidural analgesia, $\mathbf{n}$ (\%) & $11(52.4)$ \\
\hline ICU admission, $\mathbf{n}$ (\%) & $11(52.4)$ \\
\hline
\end{tabular}

Legend

$B S A$, body surface area ; BMI, body mass index; $A S A$, American Society of Anesthesiologists; ICU, Intensive Care Unit . 